\title{
Measurement of Microclimate within Clothing Using the Combination Technique of Infrared Ray Absorption Method And Holographic Interferometry
}

\author{
Takako Ninagawa $^{1}$, Tadashi Konishi ${ }^{*}, 2$ and Akira Narumi ${ }^{1}$ \\ ${ }^{1}$ Kanagawa Institute of Technology, 1030 Shimo-Ogino, Atsugi 243-0292, Japan \\ ${ }^{2}$ Oita National College of Technology, 1666 Maki, Oita 870-0152, Japan
}

\begin{abstract}
The wear comfort depends on the condition of micro-space between cloth and skin of human body. Heat and mass transfer occurs through clothing due to heat and sweat evaporation release from human body in this space. This paper applied a new measurement technique that combines infrared ray absorption method and holographic interferometry to this space to obtain the transient temperature and concentration distributions without and within cloth. The porous films with known specification were used for cloth to verify the usefulness of this technique. The measuring results were visualized by superimposing infrared ray image and interferogram to understand easily the passing behaviors of vapor through cloth. The calculated results of temperature and concentration distributions showed some significant differences in the permeation behaviors of vapor due to film. They fundamentally agreed with the property of film. As a result, this measurement technique was proved to be useful for microclimate within and without clothing.
\end{abstract}

Keywords: Infrared ray absorption, holographic interferometry, transient temperature and concentration distributions, filmpermeation of vapor.

\section{INTRODUCTION}

The wear comfort of clothing depends on the condition of the narrow space between skin of human body and clothing. This space is widely called as microclimate within clothing [1]. There occurs heat and mass transfer through cloth due to heat and sweat evaporation release from human body. This schematic diagram is shown in Fig. (1). To clarify the relationship between the wear comfort and microclimate within clothing, it is quite essential to understand the passing process of sweat and heat release through cloth. However, there is no available measurement technique for transient simultaneous temperature and concentration distributions. This paper applied the new technique [2] of combining infrared ray absorption method with real time holographic interferometry to microclimate within and without cloth, and discussed its usefulness by evaluating passing characteristic of heat and vapor through cloth from the obtained results.

\section{EXPERIMENTAL APPARATUS AND PROCEDURE}

This measurement technique is characterized by combining infrared ray (IR) and absorption method with real time holographic interferometry (HI). The principles [2-7] of this measurement technique depend on the attenuation of radiation intensity of IR due to the vapor absorption and the variation of refractive index of phase object due to the

*Address correspondence to this author at the Department of Mechanical Engineering, Oita National College of Technology, Address: 1666 Maki Oita, 870-0152, Japan; Tel: +81-97-552-6921; Fax: +81-97-552-6975;

E-mail: konishi@oita-ct.ac.jp

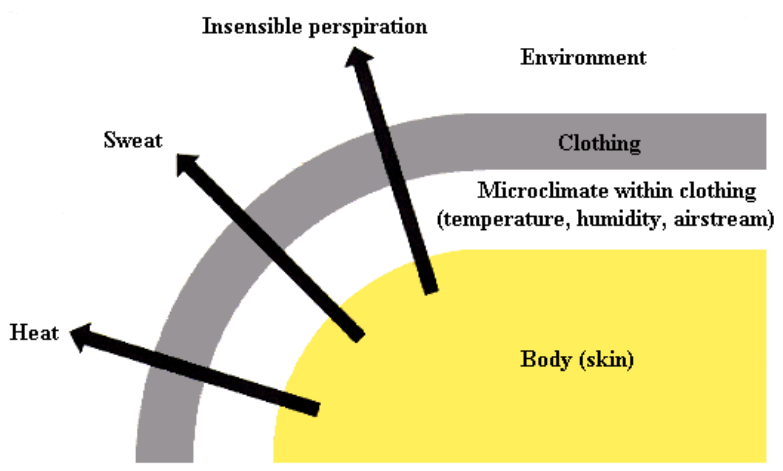

Fig. (1). Schematic Diagram of Microclimate Within Clothing [1].

changes of temperature and concentration. The concentration value can be obtained at any local point from LambertBeer's law through IR absorption method. The value of temperature is calculated by substituting this concentration with the Lorentz-Lorentz equation.

The optical setup used in this paper is shown in Fig. (2). We set the isothermally heated black-painted copper plate at 80 degree Celsius to emit IR. The radiation intensity was measured with IR camera having the resolution of $12 \mathrm{bit}$. The absorption coefficient was determined by volume percent of the saturated vapor at liquid temperature, and the temperature ratio was measured with IR camera without liquid. He-Ne gas laser with $30 \mathrm{~mW}$ was used for the light source of HI. The test section is shown in. Fig. (3). The test section has a three-storied structure. Bottom part is used to 
Table 1. Specification of Film Used in this Research

\begin{tabular}{|l|c|c|c|c|c|}
\hline Film & 1 & 2 & 3 & 4 & 5 \\
\hline Porosity $\boldsymbol{\varphi}[\boldsymbol{\%}]$ & 26 & 26 & 26 & 68 & 43 \\
\hline Thickness $\mathbf{t}[\boldsymbol{\mu m}]$ & 100 & 300 & 500 & 108 & 105 \\
\hline $\begin{array}{l}\text { Diameter of opening } \mathbf{d} \\
(\boldsymbol{\mu} \mathrm{m})\end{array}$ & 17 & 17 & 17 & $=<1$ & $=<1$ \\
\hline $\begin{array}{l}\text { Permeability of air } \\
\boldsymbol{\tau}(\mathbf{s e c} / \mathbf{1 0 0 c c})\end{array}$ & 1400 & 1400 & 1400 & 720 & 20000 \\
\hline
\end{tabular}

keep liquid temperature constant by circulating brine from the constant temperature bath. Middle part is the liquid container having $60 \mathrm{~mm}$ of width, $160 \mathrm{~mm}$ of length and $5 \mathrm{~mm}$ of depth. Upper part is the measuring region. Shutter was mounted $1 \mathrm{~mm}$ upward from liquid surface in the vertical direction. Methanol was used for spontaneously evaporating liquid. Liquid temperature was set at about $40^{\circ} \mathrm{C}$. The main purpose of this paper is to verify this technique's usefulness for microclimate within clothing. Therefore, we used the porous film of clear specification rather than the cloth, such as cotton and nylon etc. which can easily be deformed under great load. The specification of porous films used in this paper is shown in Table $\mathbf{1}$.

\section{RESULTS AND DISCUSSIONS}

\subsection{Measuring Accuracy}

We discuss the measuring accuracy of this measurement technique using the results of the case having no cloth. The concentration distribution obtained by IR camera was compared with that obtained by substituting temperature measured by thermocouples ( $1 \mathrm{~mm}$ interval in the vertical direction from the shutter) for Lorentz-Lorentz equation. The temperature distribution obtained by substituting the concentration measured by IR camera for Lorentz-Lorentz equation was compared with that measured by thermocouples. Fig. (4) shows typical distribution profiles of mole fraction and temperature along the vertical direction at the center of the width of the test section. In Fig. (4a), the blue solid circles stand for the concentration distribution obtained only by IR camera. On the other hand, red one stands for the concentration distribution calculated by both temperature change, which is measured by thermocouples, and the holographic interferometry fringe shifts (6 fringes are appeared), which are caused by both concentration and temperature change. In Fig. (4b), the red solid circles stand

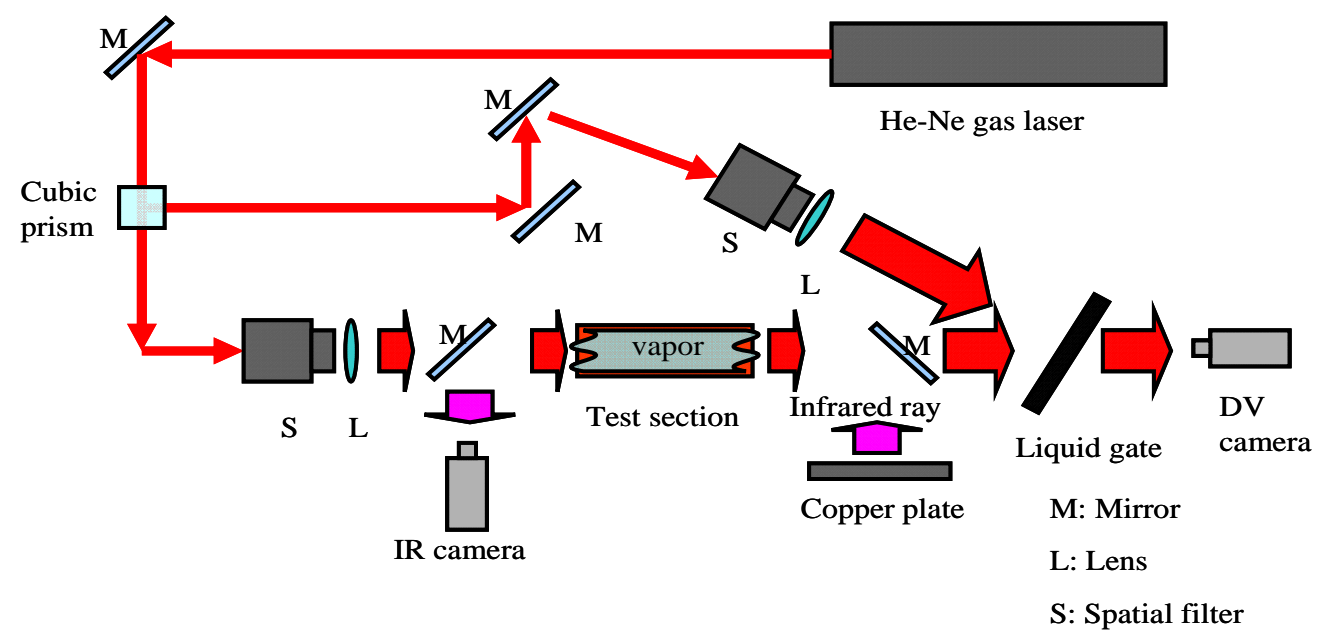

Fig. (2). Optical setup.

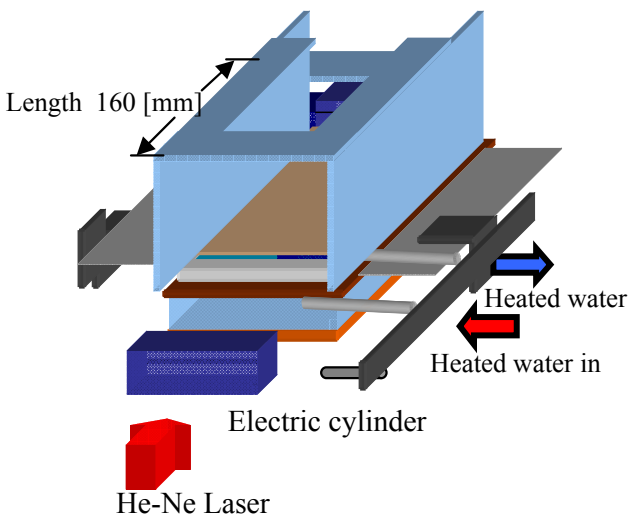

(a) Schematic Diagram of Test Section

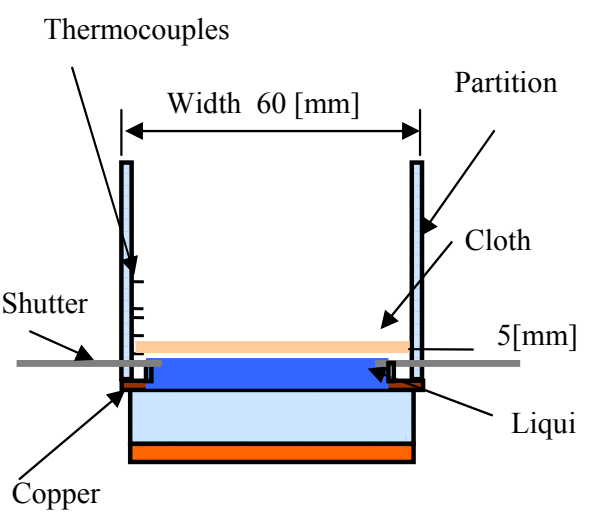

(b) Front view of test section

Fig. (3). Test section. 
for the temperature distribution obtained only by thermocouples. The blue solid circles stand for the temperature distribution calculated by both concentration change, which is measured by IR camera, and holographic interferometry fringe shifts. Both results agree to each other within $10 \%$ at maximum. Thus we confirm that this measurement technique is capable of measuring microclimate within cloth with satisfactory accuracy.

\subsection{Visualization by Superimposed Image of HI and IR}

We made the superimposed image of IR and HI images to visualize the permeating behaviors of vapor through cloth. The method of making superimposed images is shown in Fig. (5). Fig. (6) shows the superimposed images of HI and IR with the time progress after the opening of shutter in the cases of porosity $\varphi=0.68$ and 0.43 , respectively. These images clearly show that more vapor permeates through film in the case of porosity $\varphi=0.68$ than in $\varphi=0.643$. This fact suggests that the superimposed image is effective to understand the film-permeation behaviors of vapor easily.

\subsection{Transient Concentration and Temperature Distributions}

\subsubsection{The Difference Due to the Property of Film}

Figs. (7-10) show the transition of concentration and temperature distributions along the vertical line at the center of the width of test section calculated from IR and HI images with time progress after the opening of shutter. As vapor passes through cloth fluctuation due to air in the upper region of cloth has been observed on a large scale. The distributions after $10 \mathrm{~s}$ specifically at $30 \mathrm{~s}$ were found to be strongly affected by this air fluctuation which should be taken into account.

The difference in temperature and concentration distributions due to thickness of film is showed in Figs. (7, 8). Here, the results at $30 \mathrm{~s}$ were not used for the above-

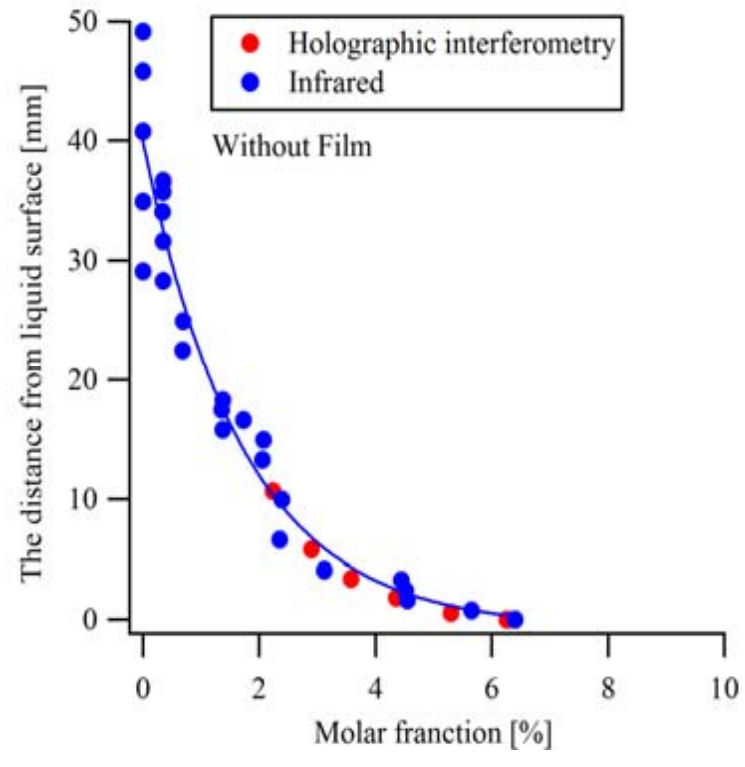

(a) Concentration distribution mentioned reason. The shape of concentration distribution in the upper region of the film is showed in Fig. (7). The shape of the case of $\mathrm{t}=500 \mu \mathrm{s}$ is steeper than that in the case of $\mathrm{t}=100 \mu \mathrm{m}$. with the increase of distance from the film. Fig. (8) suggests that the heat transfer is slightly extended to the upper region in the case of film 1 than in the case of film 3 . From these results, it was found that vapor passes more easily through film due to its lesser thickness, but its difference is minor.

We next discuss the difference in concentration and temperature distributions due to porosity by comparing Figs. $(9,10)$. The shape of concentration distribution suggests that film 5 blocks the permeation of vapor more strongly than film 4. It should be noted that there is also the difference in permeability of air as well as in porosity between these two films. Here, permeability of air means the time taken by the gas of $100 \mathrm{cc}$ to permeate through film completely. This value of film 5 is 30 times more difficult to permeate through film for gas as compared to film 4 (see Table 1).

\subsubsection{Evaluation of Ease of Vapor's Film-Permeation}

We try to estimate the degree of ease for vapor to permeate through film using the difference in concentration at both sides of film. Fig. (11) shows the results that these values can to be divided into 3 groups by permeability of air shown in Table 1. This fact suggests that with lesser thickness or with small a opening diameter the effect of permeability is very significant.

\section{CONCLUSIONS}

This paper applied a new measurement technique of combination of infrared ray absorption method and holographic interferometry that we have developed to the microclimate within clothing to obtain the transient temperature and concentration distributions simultaneously. The porous films known specification were used for cloth

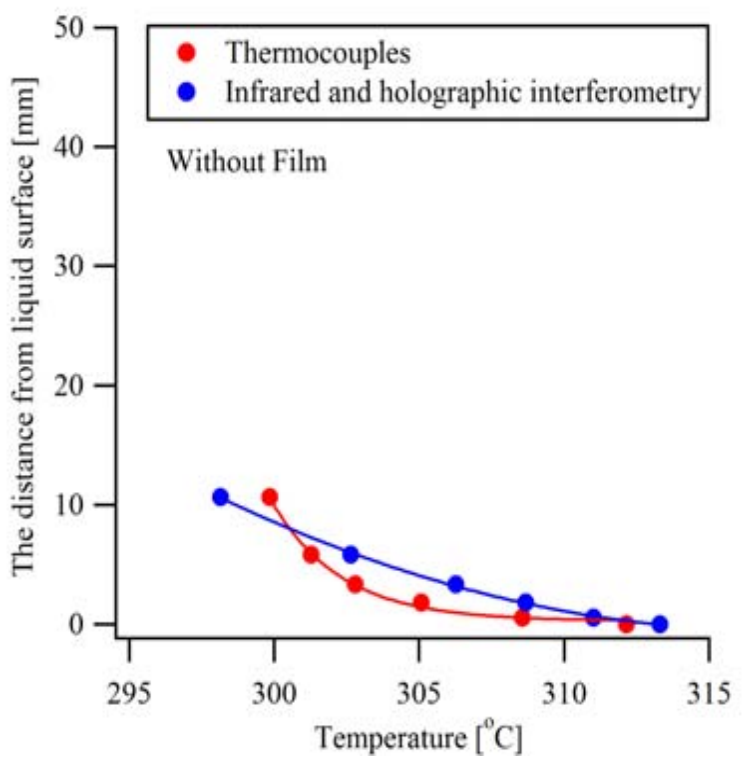

(b) Temperature distribution

Fig. (4). The measuring accuracy of this technique. 


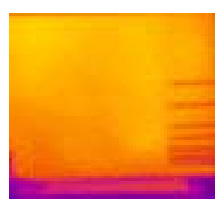

IR original
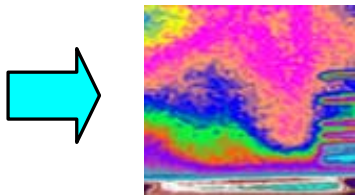

IR colored

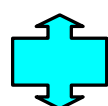

$\square$

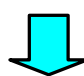

HI image
High

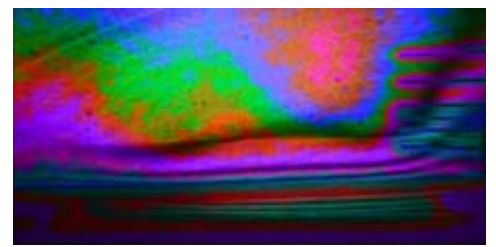

Superimposed image

Fig. (5). Making of superimposed image of IR and HI.

Film5 $(\varphi=68 \%)$
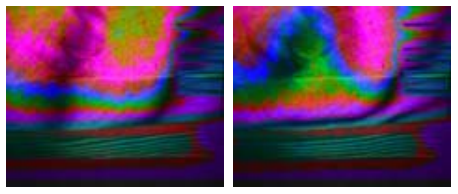

Film6 $(\varphi=43 \%)$
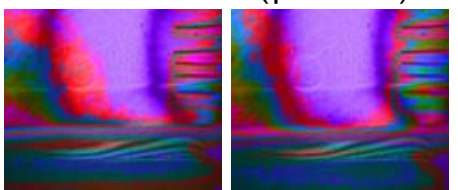

$1 \mathrm{sec}$

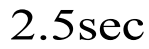

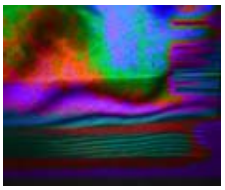

Liquid Surface
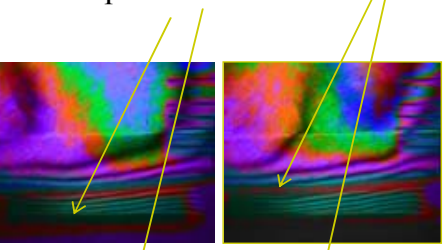

HIGH
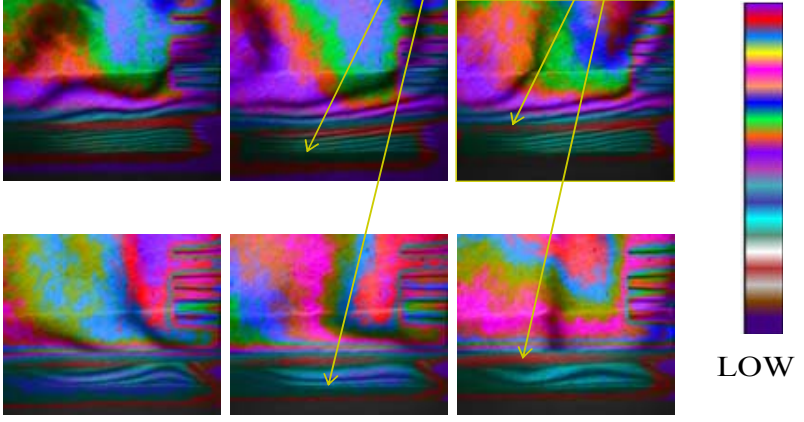

$5 \mathrm{sec}$

10 sec

$30 \mathrm{sec}$

Fig. (6). Visualization of transient of passing behaviors of vapor in terms of superimposed images.

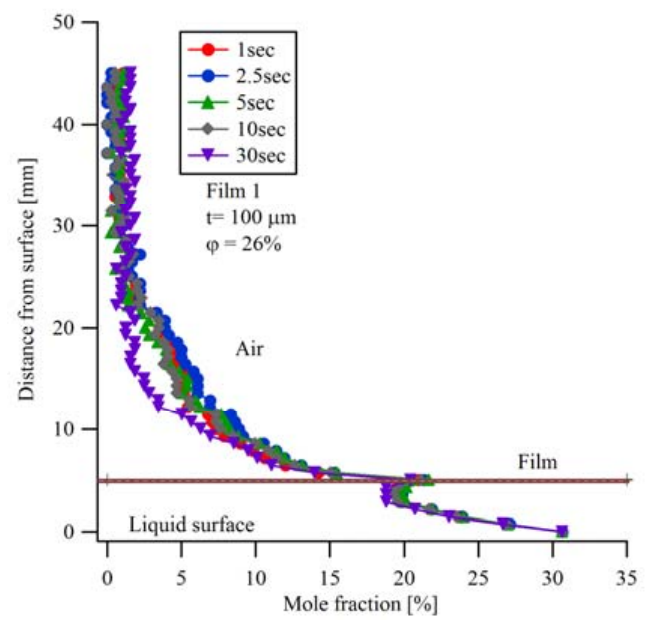

(a) Film 1

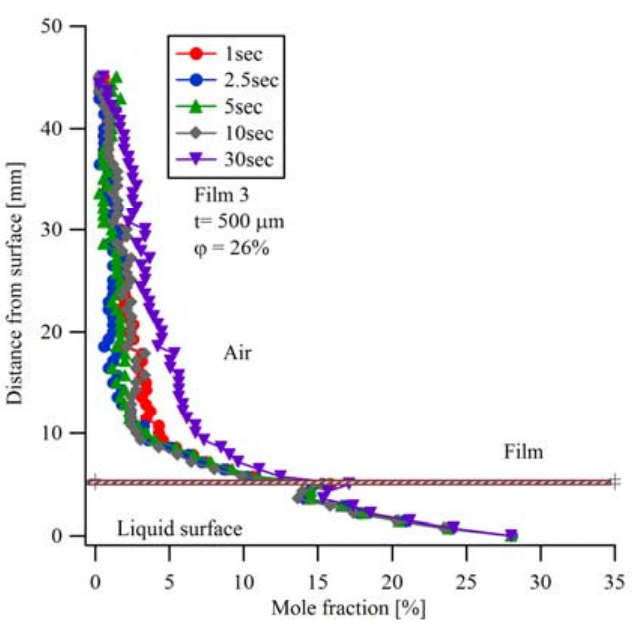

(b) Film 3

Fig. (7). Difference in concentration distribution due to thickness.

instead of cloth to verify the usefulness of this technique. Though the obtained results showed a few contradictions they fundamentally corresponded to the property of film.
Consequently, it was proved by this paper that this measurement technique is quite useful for microclimate within and without clothing. 


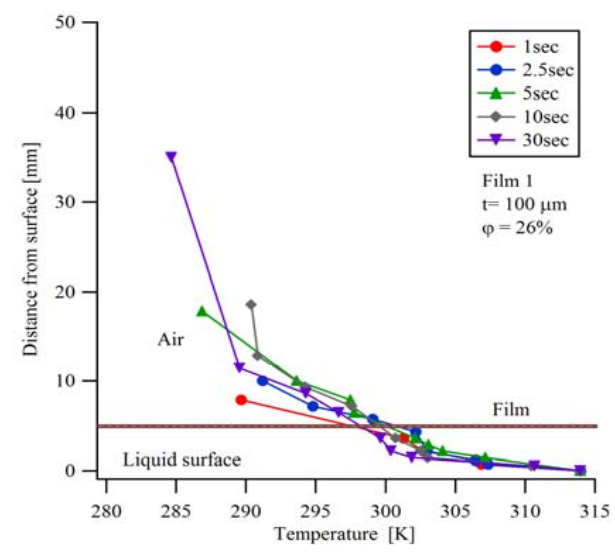

(a) Film 1

Fig. (8). Difference in temperature distribution due to thickness.

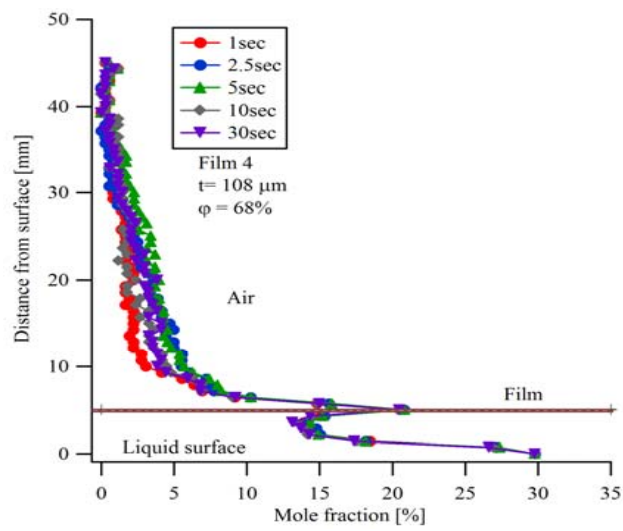

(a) Film 4

Fig. (9). Difference in concentration distribution due to porosity.

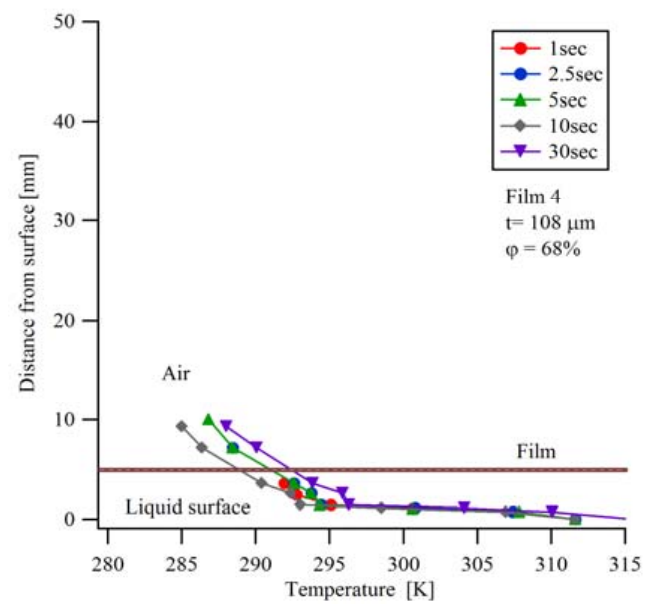

(a) Film 4

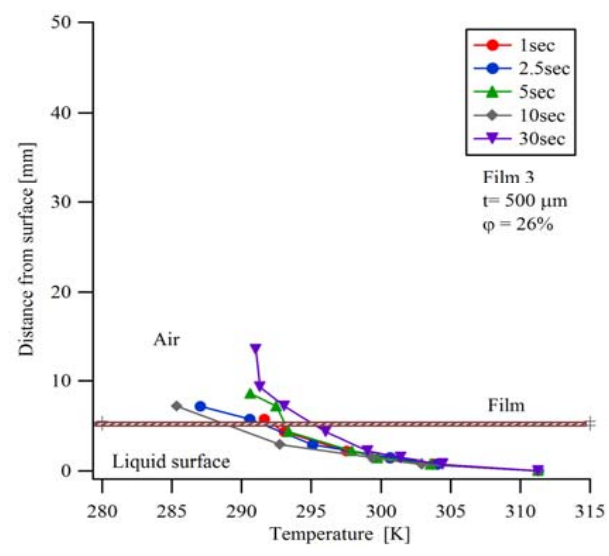

(b) Film 3

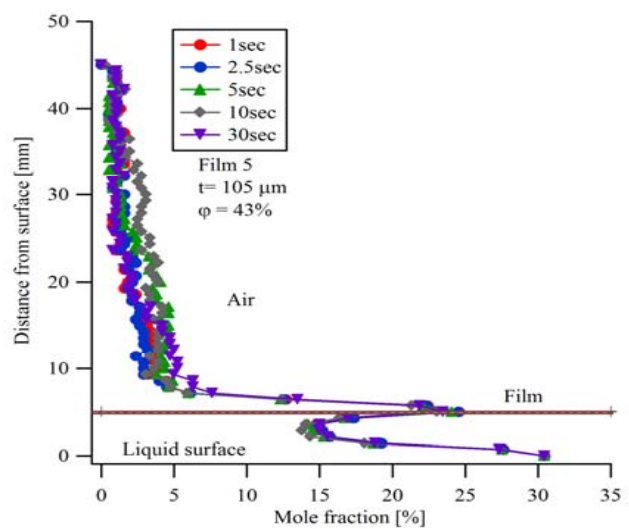

(b) Film 5

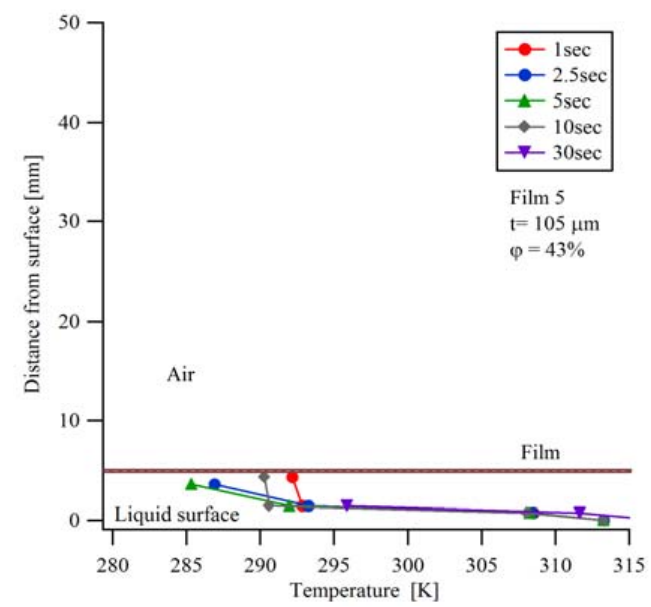

(b) Film 5

Fig. (10). Difference in temperature distribution due to porosity. 


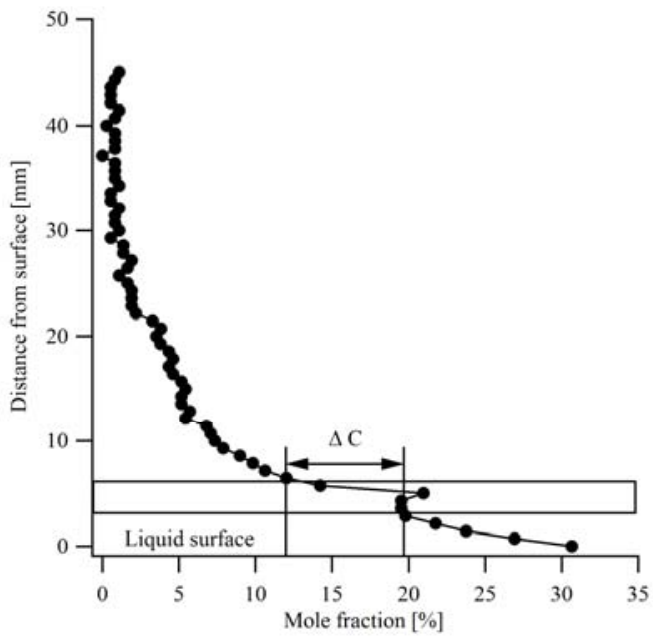

(a) Definition of difference in concentration $\Delta \mathrm{C}$ at both sides of film

Fig. (11). Permeation degree of vapor due to film.

\section{CONFLICT OF INTEREST}

The authors confirm that this article content has no conflict of interest.

\section{ACKNOWLEDGEMENTS}

We appreciate that this paper is supported by funds of Grant-in-Aid for Scientific Research No. 24650474 in Japan.

\section{REFERENCES}

[1] Toyobo's Science in Comfort. Home Page of Toyobo. Available from: http://www.toyobo.co.jp/e/seihin/sports/ifukunai/ ifuku

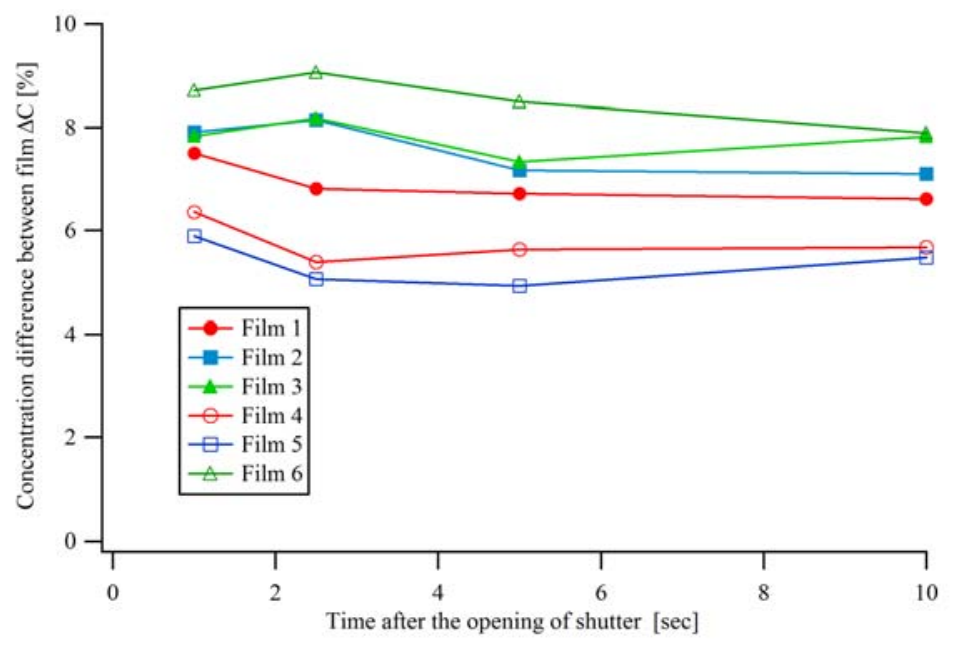

(b) The change of difference in concentration $\Delta \mathrm{C}$ with time

[2] Konishi T, Naka S, Ito A, Saito K. Transient two-dimensional fuelconcentration measuarement technique. Appl Opt 1997; 36(33): 8815-9.

[3] Konishi T, Ito A, Kudo Y. Simultaneous measurement of temperature and chemical species concentrations with holographic interferometer and infrared absorption. Appl Opt 2006; 45(22): 5725-32.

[4] Mayinger F, Panknin W. Holography in heat and mass transfer. Proc 5th Int Heat Transfer Conf 1974; vol. 1: pp. 28-43.

[5] Atkins PW. Physical Chemistry. New York: Freeman 1994

[6] Vest CM. Holographic Interferometry. New York: John Wiley \& Sons 1979.

[7] Gardiner WC, Hidaka Y Jr, Tanzawa T. Refractivity of combustion gases. Combustion Flame 1981; 40; 213-9. 